\title{
HAMILTONIAN EQUATIONS FOR A TANGENT STRUCTURE ON TWISTOR SPACE
}

ZEKI KASAP

\author{
Department of Elementary Mathematics Education \\ Faculty of Education \\ Pamukkale University \\ Kinikli Campus \\ Denizli \\ Turkey \\ e-mail: zekikasap@hotmail.com
}

\begin{abstract}
The paper purpose to introduce some partial differential equations on twistor space, with an emphasis on Hamilton equations. Classical mechanics has provided effective solution methods for dynamic systems. Hamilton equation is one of these methods and it is a model that shows the movement over time of dynamic systems. Twisted geometries are discrete geometries that plays a role in loop quantum gravity and spin foam models, where they appear in the semiclassical limit of spin networks. It is well-known that twistor spaces are certain complex 3-manifolds, which are associated with special conformal Riemannian geometries on 4-manifolds. This correspondence between complex 3-manifolds and real 4-manifolds is called the Penrose twistor correspondence. In this study, showing motion modelling partial differential equations have been obtained for movement of objects in space and solutions of these equations have been generated by using the Maple software. Additionally, the implicit solution of the equation obtained as a result of a special selection of graphics to be drawn.
\end{abstract}

2010 Mathematics Subject Classification: 14D21, 34N05, 53C15, 70Q05, 70 S05.

Keywords and phrases: twistor, Kählerian manifold, mechanical system, dynamic equation, almost complex, Hamiltonian formalism.

Received November 5, 2015

(C) 2015 Scientific Advances Publishers 


\section{Introduction}

Twistor theory maps the geometric objects of conventional $3+1$ space-time (Minkowski space) into geometric objects in a 4-dimensional space endowed with a Hermitian form of signature $(2,2)$. This space is called twistor space, and its complex valued coordinates are called "twisters". Twistors are essentially complex objects and in order to proceed we shall have to consider the complexification of the compactified Minkowski space. Penrose introduced twistor theory [1]. Penrose created the twistor theory to solve problems in mathematical physics [2]. Pantilie shown that a natural class of twistorial maps gives a pattern for apparently different geometric maps, such as $(1,1)$-geodesic immersions from (1, 2)-symplectic almost Hermitian manifolds and pseudo horizontally conformal submersions with totally geodesic fibres for which the associated almost CR-structure is integrable [3]. Ianus et al. defined a natural notion of quaternionic map between almost quaternionic manifolds [4]. Cecotti et al. examined twistorial topological strings by considering $t t^{*}$ geometry of the $4 d N=2$ supersymmetric theories on the Nekrasov-Shatashvili $\frac{1}{2} \Omega$ background [5]. Santa-Cruz examined the hyper-Kähler geometry of complex adjoint orbits from the point of view of twistor theory [6]. Albuquerque used twistor theory to describe virtually new constructions of Hermitian and quaternionic Kähler structures on tangent bundles [7]. Davidov and Mushkarov introduced that the twistor construction is applied for obtaining examples of generalized complex structures [8]. Loubeau and Pantilie presented that Weyl spaces provide a natural context for harmonic morphisms [9]. Davidov and Mushkarov presented that the twistor method is applied for obtaining examples of generalized Kähler structures, which are not yielded by Kähler structures [10]. Dunajski introduced an elementary and self-contained review of twistor theory as a geometric tool for solving non-linear differential equations [11]. Marchiafava revealed that an alternative 
proof of a characterization of twistorial maps between quaternionic projective spaces [12]. Tekkoyun obtained Euler-Lagrange and Hamiltonian equations on $\mathbb{R}_{n}^{2 n}$, which is a model of para-Kählerian manifolds of constant $J$-sectional curvature [13].

\section{Preliminaries}

Definition 1. A Hermitian form on a vector space $V$ over the complex field $\mathbb{C}$ is a function $f: V \times V \rightarrow \mathbb{C}$ such that for all $u, v, w$ in $V$ and all $a, b$ in $\mathbb{R}$, (1) $f(a u+b v, w)=a f(u, w)+b f(v, w)$. (2) $f(u, v)=\overline{f(u, v)}$. Here, the bar indicates the complex conjugate. It follows that $f(u, a v, b w)=\bar{a} f(u, v)+\bar{b} f(u, w)$, which can be expressed by saying that $f$ is antilinear on the second coordinate.

Definition 2. Let $\vec{X}=\left(x_{i}\right), \vec{Y}=\left(y_{i}\right) \in \mathbb{R}^{3}$ be any two vectors. As follows:

$$
<,>: \mathbb{R}^{3} \times \mathbb{R}^{3} \rightarrow \mathbb{R}^{1}, \quad<\vec{X}, \vec{Y}>_{L}=-x_{1} y_{1}+x_{2} y_{2}+x_{3} y_{3},
$$

in the form of a function. This function are bilinear and symmetric. This the inner product function $\langle\vec{X}, \vec{Y}\rangle_{L}$ along with $\mathbb{R}^{3}$ is called Minkowski space or the Lorentz space and it is been shown $\mathbb{R}_{1}^{3}$.

Theorem 1. Let $X \in \mathbb{R}_{1}^{3}$ be any one vector.

(1) If $<\vec{X}, \vec{X}>_{L}>0$ or $\vec{X}=\overrightarrow{0}, \vec{X}$ is spacelike;

(2) If $<\vec{X}, \vec{X}>_{L}<0, \vec{X}$ is timelike;

(3) If $<\vec{X}, \vec{X}>_{L}<0, \vec{X}$ is lightlike (isotropic, null). 
Definition 3. Let $V$ be a vector space over $\mathbb{R}$. Let $M$ be a differentiable manifold of dimension $2 n$, and suppose $J$ is a differentiable vector bundle isomorphism $J_{x}: T_{x} M \rightarrow T_{x} M$.

(1) A (almost) complex structure on $M$ for $J^{2} d=-I d$;

(2) A (almost) paracomplex structure on $M$ for $J^{2} d=I d$;

(3) A tangent (exact) structure on $M$ for $J^{2} d=0$,

where $J^{2}=J \circ J$, and $I$ is the identity (unit) operator on $V$ by the map $J: V \rightarrow V$ and $V=\mathbb{R}^{n} \oplus \mathbb{R}^{n}$.

Definition 4. A tangent structure $J$ on $M$ assigns to each $p \in M$ a linear map $J_{p}: T_{p} M \rightarrow T_{p} M$ that is smooth in $p$ and satisfies $J_{p}^{2} d=0$ for all $p$. The pair $(M, J)$ is called a tangent manifold.

Theorem 2. Any (para)complex manifold $M$ is also an almost (para)complex manifold.

Lemma 1. Let $M$ be a smooth manifold. If $M$ admits a complex structure $A$, then $M$ admits an almost complex structure J. Let $\operatorname{dim}_{\mathbb{C}} M=m$ and $(z, U)$ be any holomorphic chart inducing a coordinate frame $\partial x_{1}, \partial y_{1}, \ldots, \partial x_{m}, \partial y_{m}$. Then $J$ is given locally as

$$
J_{p}\left(\partial x_{i} p\right)=\partial y_{i} p, \quad J_{p}\left(\partial y_{i} p\right)=-\partial x_{i} p
$$

where $1 \leq i \leq m$ and $p \in U$ [14].

Definition 5. Let $(M, J, g)$ be a $2 n$-dimensional almost complex manifold and $g$ is a metric, i.e., $J$ is an almost complex structure such that $J^{2} X=-X, g(J X, J Y)=-g(X, Y)$, for all vector fields $X, Y$ on $M$ and $g$ is a metric. 
Definition 6. Let $M$ be a complex manifold with complex structure $J$ and compatible Riemannian metric $g=<.$, . $>$ as in $\langle J X, J Y>=<X, Y>$. The alternating 2-form $\omega(X, Y):=g(J X, Y)$ is called the associated Kähler form. We can retrieve $g$ from $\omega, g(X, Y)=\omega(X, J Y)$. We can say that $g$ is a Kähler metric and that $M$ is a Kähler manifold if $\omega$ is closed and $(M, g)$ is displayed in the form.

Definition 7. Let $M$ be a complex manifold. A Riemannian metric on $M$ is called Hermitian if it is compatible with the complex structure $J$ of $M,<J X, J Y>=<X, Y>$. Then the associated differential two-form $\omega$ defined by $\omega(X, Y)=<J X, Y>$ is called the Kähler form. It turns out that $\omega$ is closed if and only if $J$ is parallel. Then $M$ is called a Kähler manifold and the metric on $M$ a Kähler metric. Kähler manifolds are modelled on complex Euclidean space [15].

Definition 8. Suppose that $\xi$ is a vector field: that is, a vector-valued function with Cartesian coordinates $\left(\xi_{1}, \ldots, \xi_{n}\right)$; and $\mathbf{x}(t)$ a parametric curve with Cartesian coordinates $\left(x_{1}(t), \ldots, x_{n}(t)\right)$. Then $\mathbf{x}(t)$ is an integral curve of $\xi$ if it is a solution of the following autonomous system of ordinary differential equations: $\frac{d x_{1}}{d t}=\xi_{1}\left(x_{1}, \ldots, x_{n}\right), \ldots, \frac{d x_{n}}{d t}=\xi_{n}\left(x_{1}, \ldots, x_{n}\right)$. Such a system may be written as a single vector equation

$$
\xi(\mathbf{x}(t))=\mathbf{x}^{\prime}(t)=\frac{\partial}{\partial t}(\mathbf{x}(t))
$$

\section{Twistor Theory}

Twistor theory is essentially complex objects, like wave functions in quantum mechanics, as well as endowed with holomorphic and algebraic structure sufficient to encode space-time points. In this meaning, twistor space can be considered more primitive than the space-time itself and indeed provides a background against which space-time could be meaningfully quantized. It is the use of complex analytic methods to solve problems in real differential geometry. The following description is given for twister space. 
Definition 9. Minkowski space is a four-dimensional space possessing a Minkowski metric a metric tensor having the form

$$
d \tau^{2}=-\left(d x^{0}\right)^{2}+\left(d x^{1}\right)^{2}+\left(d x^{2}\right)^{2}+\left(d x^{3}\right)^{2}
$$

In Equation (5) above, the metric signature $(1,3)$ is assumed; under this assumption, Minkowski space is typically written $\mathbb{R}^{(1,3)}$.

Lemma 2. Let $V$ be a $2 n$-dimensional real vector space and $V^{*}$ its dual space. Then the vector space $V \oplus V^{*}$ admits a natural neutral metric defined by

$$
\langle X+\xi, Y+\eta\rangle=\frac{1}{2}(\xi(Y)+\eta(X)), \quad X, Y \in V \text { and } \xi, \eta \in V^{*}
$$

Lemma 3. Let $V$ be a 2-dimensional real vector space and let $\left\{Q_{i}=e_{i}+\eta_{i}\right\}, 1 \leq i \leq 4$, be an orthonormal basis of the space $V \oplus V^{*}$ endowed with its natural neutral metric (6). Then

$$
e_{3}=a_{11} e_{1}+a_{12} e_{2}, \quad e_{4}=a_{21} e_{1}+a_{22} e_{2}
$$

where $A=\left[a_{i j}\right]$ is an orthogonal matrix. If $\operatorname{det} A=1$, the basis $\left\{Q_{i}\right\}$ yields the canonical orientation of $V \oplus V^{*}$ and if $\operatorname{det} A=-1$ it yields the opposite one (proof see [8]).

Theorem 3. Let $V$ be a 2-dimensional real vector space. Take a basis $\left\{e_{1}, e_{2}\right\}$ of $V$ and let $\left\{\eta_{1}, \eta_{2}\right\}$ be its dual basis. Then $\left\{Q_{1}=e_{1}+\eta_{1}\right.$, $\left.Q_{2}=e_{2}+\eta_{2}, Q_{3}=e_{3}-\eta_{3}, Q_{4}=e_{4}-\eta_{4}\right\}$ is an orthonormal basis of $V \otimes V^{*}$ with respect to the natural neutral metric (5) and is positively oriented with respect to the canonical orientation of $V \otimes V^{*}$. Put $\varepsilon_{k}=\left\|Q_{k}\right\|^{2}, \quad k=1, \ldots, 4$, and define skew-symmetric endomorphisms of $V \otimes V^{*} \quad$ setting $S_{i j} Q_{k}=\varepsilon_{k}\left(\delta_{i k} Q_{j}-\delta_{i k} Q\right), 1 \leq i, j, k \leq 4$. Then the endomorphisms 


$$
\begin{aligned}
& I_{1}=S_{12}-S_{34}, \quad J_{1}=S_{12}+S_{34}, \\
& I_{2}=S_{13}-S_{24}, \quad J_{2}=S_{13}+S_{24}, \\
& I_{3}=S_{14}-S_{23}, \quad J_{3}=S_{14}-S_{23},
\end{aligned}
$$

constitute a basis of the space of skew-symmetric endomorphisms of $V \otimes V^{*}$. Let $I \in G^{+}(V)$ and $J \in G^{-}(V) . \quad I_{1}^{2}=I_{2}^{2}=I d, I_{3}^{2}=-I d$, $J_{1}^{2}=J_{2}^{2}=I d, J_{3}^{2}=-I d, I_{r} I_{s}=-I_{s} I_{r}, J_{r} J_{s}=-J_{s} J_{r}, 1 \leqslant r \neq s \leqslant 3$, and $I_{r} J_{s}=J_{s} I_{r}, 1 \leqslant r, s \leqslant 3$. Evaluating the latter identity at $e_{1}, \ldots, e_{4}$. It follows that

$$
\begin{aligned}
& I e_{1}=x_{2} e_{1}+\left(x_{1}+x_{3}\right) e_{2}, \quad J e_{1}=y_{2} e_{1}+\left(y_{1}-y_{3}\right) \eta_{2}, \\
& I e_{2}=-\left(x_{1}-x_{3}\right) e_{1}-x_{2} e_{2}, \quad J e_{2}=y_{2} e_{2}-\left(y_{1}-y_{3}\right) \eta_{1}, \\
& I \eta_{1}=-x_{2} \eta_{1}+\left(x_{1}-x_{3}\right) \eta_{2}, \quad J \eta_{1}=\left(y_{1}+y_{3}\right) e_{1}-y_{2} \eta_{2}, \\
& I \eta_{2}=-\left(x_{1}+x_{3}\right) \eta_{1}+x_{2} \eta_{2}, \quad J \eta_{2}=-\left(y_{1}+y_{3}\right) e_{1}-y_{2} \eta_{2} .
\end{aligned}
$$

Let $\Psi=\sum_{r=1}^{3}\left(x_{r} I_{r}+y_{r} J_{r}\right) \quad$ be a complex structure on $V \otimes V^{*}$ compatible with the metric. Then we have $x_{1}^{2}+x_{2}^{2}-x_{3}^{2}=0$ and $y_{1}^{2}+y_{2}^{2}-y_{3}^{2}=0$ for $\Psi^{2}=0[8]$.

Proof. Let us set $\Psi=\sum_{r=1}^{3}\left(x_{r} I_{r}+y_{r} J_{r}\right)$. Let's we have account $\Psi^{2}$ using Definition 4.

$$
\begin{aligned}
\Psi^{2}=\sum_{r=1}^{3}\left(x_{r} I_{r}+y_{r} J_{r}\right)^{2}= & \left(x_{1}^{2}+x_{2}^{2}-x_{3}^{2}+y_{1}^{2}+y_{2}^{2}-y_{3}^{2}\right) I d \\
& +2 \sum_{\tau, s=1}^{3}\left(x_{r} y_{s}+I_{r} J_{s}\right) .
\end{aligned}
$$

We see that $x_{1}^{2}+x_{2}^{2}-x_{3}^{2}+y_{1}^{2}+y_{2}^{2}-y_{3}^{2}=0, x_{r} y_{s}=0, r, s=1,2,3$.

Therefore $\Psi^{2}=0$ if and only if either 


$$
\begin{aligned}
& I=\sum_{r} x_{r} I_{r} \text { with } x_{1}^{2}+x_{2}^{2}-x_{3}^{2}=0, \\
& J=\sum_{s} y_{s} J_{s} \text { with } y_{1}^{2}+y_{2}^{2}-y_{3}^{2}=0 .
\end{aligned}
$$

This shows that the restriction of $I$ to $V$ is a complex structure on $V$ inducing the generalized complex structure. In contrast, the generalized complex structure $J$ is not induced by a complex structure or a symplectic form on $V$ [8].

Theorem 4. If $\alpha$ and $\beta$ are 1-forms, then $\alpha \wedge \beta$ is a 2-forms.

Definition 10. In three dimensions, the vector from the origin to the point with Cartesian coordinates $(x, y, z)$ can be written as [16]:

$$
r=x \vec{i}+y \vec{j}+z \vec{k}=x\left(\frac{\partial}{\partial x}\right)+y\left(\frac{\partial}{\partial y}\right)+z\left(\frac{\partial}{\partial z}\right) .
$$

Proposition 1. Let $\vec{X}, \vec{Y} \in \mathbb{R}_{1}^{3}$ be any two vector that are $\vec{X}=(x, y$ $+z, 0), \vec{Y}=(y-z,-x, 0)$. Let us consider the structure (9) using the holomorphic property at (8). We can define the following holomorphic structure with $e_{1}=\frac{\partial}{\partial x}, e_{2}=\frac{\partial}{\partial y}, e_{3}=\frac{\partial}{\partial z}$,

$$
\begin{aligned}
& \text { (1) } J \frac{\partial}{\partial x}=x \frac{\partial}{\partial x}+(y+z) \frac{\partial}{\partial y} \\
& \text { (2) } J \frac{\partial}{\partial y}=(y-z) \frac{\partial}{\partial x}-x \frac{\partial}{\partial y} \\
& \text { (3) } J \frac{\partial}{\partial z}=0
\end{aligned}
$$

Dual form $\left(J^{*}\right)$ of above holomorphic structure (13) is as follows:

(1) $J^{*}(d x)=x d x+(y+z) d y$,

(2) $J^{*}(d y)=(y-z) d x-x d y$,

(3) $J^{*}(d z)=0$. 
Now, we denote the structure (14) of the holomorphic property:

(1) $J^{* 2}(d x)=J^{*}(x d x+(y+z) d y)=x(x d x+(y+z) d y)+(y+z)((y-z)$ $d x-x d y)=\left(x^{2}+y^{2}-z^{2}\right) d x$,

(2) $J^{* 2}(d y)=J^{*}((y-z) d x-x d y)=(y-z)(x d x+(y+z) d y)-x((y-z)$ $d x-x d y)=\left(x^{2}+y^{2}-z^{2}\right) d y$,

(3) $J^{* 2}(d z)=0$.

As shown above, the structures (15) are tangent and $z^{2}=x^{2}+y^{2}$ for $J^{* 2}=0$. This is a cone and the graph is as follows:

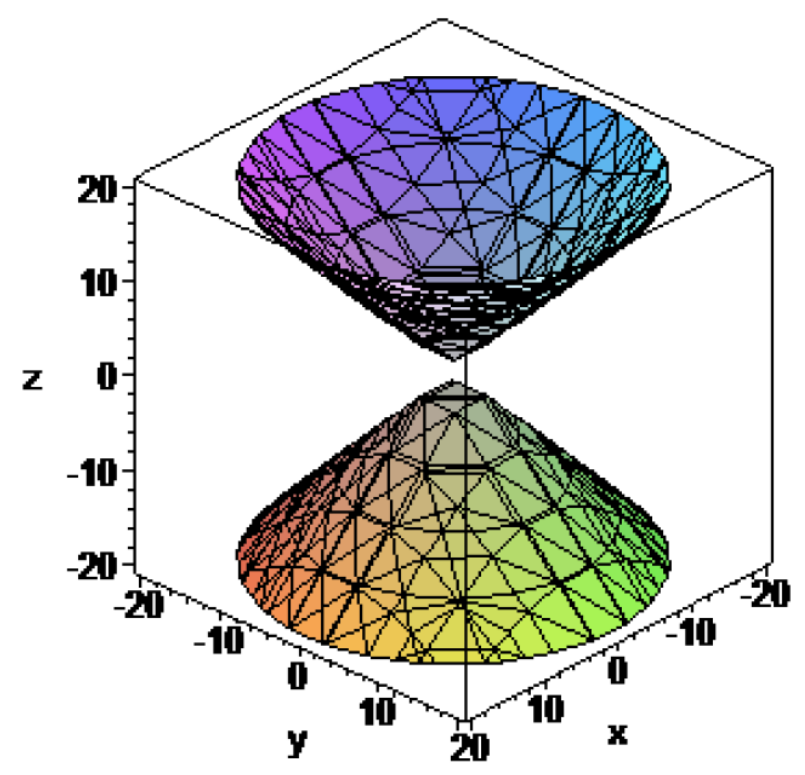

\section{Hamiltonian Mechanical Systems}

Classical mechanic is one of the major subfields for dynamical (mechanical) system. A dynamical system has a state determined by a collection of real numbers, or more generally by a set of points in an appropriate state space and it is a smooth action of the real or the integers on another object that it named usually a manifold. When the 
reals are acting, the mechanical system is called a continuous dynamical system, and when the integers are acting, the system is called a discrete dynamical system. At any given time, a dynamical system has a state given by a set of real numbers (a vector) that can be represented by a point in an appropriate state space. Dynamical systems theory is an area of mathematics used to describe the behaviour of complex dynamical systems, usually by employing differential equations or difference equations. The above-mentioned differential equations are found using the following ways:

Lemma 4. The closed 2-form on a vector field and 1-form reduction function on the phase space defined of a mechanical system is equal to the differential of the energy function 1-form of the Lagrangian and the Hamiltonian mechanical systems [17, 18].

Definition 11. Let $M$ is the configuration manifold and its cotangent manifold $T^{*} M$. By a symplectic form we mean a 2 -form $\Phi$ on $T^{*} M$ such that :

(i) $\Phi$ is closed, that is, $d \Phi=0$; (ii) for each $z \in T^{*} M, \Phi: T^{*} M \times T^{*}$ $M \rightarrow \mathbb{R}$ is weakly nondegenerate. If $\Phi_{z}$ in (ii) is nondegenerate, we speak of a strong symplectic form. If (ii) is dropped we refer to $\Phi$ as a presymplectic form. Let $\left(T^{*} M, \Phi\right)$ be a symplectic manifold. A vector field $X_{H}: T^{*} M \rightarrow T^{*} M$ is called Hamiltonian if there is a $C^{1}$ function $H: T^{*} M \rightarrow \mathbb{R}$ such that dynamical equation is determined by

$$
i_{X_{H}} \Phi=d H
$$

We say that $X_{H}$ is locally Hamiltonian vector field if $i_{X_{H}} \Phi$ is closed and where $\Phi$ shows the canonical symplectic form so that $\Phi=-d \Omega, \Omega=J^{*}(\omega)$, $J^{*}$ a dual of $J, \omega$ a 1 -form on $T^{*} M$. The trio $\left(T^{*} M, \Phi, X_{H}\right)$ is named Hamiltonian system which it is defined on the cotangent bundle $T^{*} M$ $[19,20]$. 
Definition 12. The vector field $X$ on $T^{*} M$ given by $i_{X} \omega=d H$ is called the geodesic flow of the metric $g$.

Definition 13. If $\gamma:(a, b) \rightarrow T^{*} M$ is an integral curve of the geodesic flow, then the curve $p(\gamma)$ in $M$ is called a geodesic.

The Hamiltonian of a system is defined to be the sum of the kinetic and potential energies expressed as a function of positions and their conjugate momenta. Recall from elementary physics that momentum of a particle, $p_{i}$, is defined in terms of its velocity $\dot{q}_{i}$ by $p_{i}=m_{i} \dot{q}_{i}$. In fact, the more general definition of conjugate momentum, valid for any set of coordinates, is given in terms of the Lagrangian: $p_{i}=\partial L / \partial \dot{q}_{i}$, $\dot{p}_{i}=\partial L / \partial q_{i}$. Note that these two definitions are equivalent for Cartesian variables. In terms of Cartesian momenta, the kinetic energy is given by $T=\sum_{i=1}^{n} p_{i}^{2} / 2 m_{i}$. Then, the Hamiltonian, which is defined to be the sum, $H=T+V$, expressed as a function of positions and momenta, will be given by $H\left(q_{i}, p_{i}\right)=\sum_{i=1}^{n} p_{i}^{2} / 2 m_{i}+V\left(q_{1}, \ldots, q_{n}\right)$, where $p=p_{1}, \ldots, p_{n}$. The function $H$ is equal to the total energy of the system. In terms of the Hamiltonian, the equations of motion of a system are given by Hamilton's equations:

$$
\dot{q}_{i}=\frac{\partial H}{\partial p_{i}}, \quad \dot{p}_{i}=-\frac{\partial H}{\partial q_{i}} .
$$

The solution of Hamilton's equations of motion will yield a trajectory in terms of positions and momenta as functions of time. Hamilton's equations can be easily shown to be equivalent to Newton's equations and Hamilton's equations can be used to determine the equations of motion of a system in any set of coordinates. 


\section{Hamilton Equations}

Hamilton equations are an efficient use of classical mechanics to solve problems using mathematical modelling. In this part, we present, using (17), Hamilton equations for quantum and classical mechanics which are constructed on twistorial generalized Kähler manifolds. It is well-known that the quantum mechanical commutation relations for momentum and angular momentum give simple commutation relation for twistors and dual twistors (14). Hence the quantization rule for twistor theory: holomorphic structure of dual twistors (14) are represented for mechanical systems.

Proposition 2. Let $\left(T^{*} M, g, J^{*}\right)$ be on twistorial generalized Kähler manifolds. Where in the calculations to be used (17). Imagine that the complex structures, a Liouville form and a 1-form on contact manifolds are indicated by $J^{*}, \Omega$ and $\omega$, respectively. Think about a 1-form such that

$$
\omega=y d x-x d y+d z .
$$

Now, we obtain the Liouville form with (14) as follows:

$$
\begin{aligned}
\Omega & =J^{*}(\omega)=y J^{*}(d x)+x J^{*}(d y)+J^{*}(d z) \\
& =\left(x^{2}-x y+x z\right) d x+\left(x^{2}+x y+x z\right) d y .
\end{aligned}
$$

It is well-known that if $\Phi$ is a closed on contact manifold $\left(T^{*} M, g, J^{*}\right)$, then $\Phi$ is also a symplectic structure on $\left(T^{*} M, g, J^{*}\right)$. Therefore, the 2 -form $\Phi$ shows the canonical symplectic form and derived from the 1 -form $\Omega$ to find to mechanical equations. In this case the 2 -form is calculated as: 


$$
\begin{aligned}
& \Phi=-d \Omega=\left(\frac{\partial}{\partial x} d x+\frac{\partial}{\partial y} d y\right)\left[\left(x^{2}-x y+x z\right) d x+\left(x^{2}-x y+x z\right) d y\right] \\
& =-\left[\begin{array}{l}
\frac{\partial\left(x^{2}-x y+x z\right)}{\partial x} d x \wedge d x+\frac{\partial\left(x^{2}+x y+x z\right)}{\partial x} d x \wedge d y+\frac{\partial\left(x^{2}-x y+x z\right)}{\partial y} d y \wedge d x \\
+\frac{\partial\left(x^{2}+x y+x z\right)}{\partial y} d y \wedge d y+\frac{\partial\left(x^{2}-x y+x z\right)}{\partial z} d z \wedge d x+\frac{\partial\left(x^{2}+x y+x z\right)}{\partial z} d z \wedge d y
\end{array}\right] \\
& =(2 x+y+z) d y \wedge d x-x d x \wedge d y+x d x \wedge d z+x d y \wedge d z
\end{aligned}
$$

Proposition 3. Let $X_{H}$ be a vector field

$$
X_{H}=X \frac{\partial}{\partial x}+Y \frac{\partial}{\partial y}+Z \frac{\partial}{\partial z}
$$

so that called to be Hamiltonian vector field associated with Hamiltonian energy $H$ and determined by (17). Then there are

$$
\begin{gathered}
\Phi\left(X_{H}\right)=(2 x+y+z) X\left[d y\left(\frac{\partial}{\partial x}\right) d x-d x\left(\frac{\partial}{\partial x}\right) d y\right]-x X\left[d x\left(\frac{\partial}{\partial x}\right) d y-d y\left(\frac{\partial}{\partial x}\right) d x\right] \\
+x X\left[d x\left(\frac{\partial}{\partial x}\right) d z-d z\left(\frac{\partial}{\partial x}\right) d x\right]+x X\left[d y\left(\frac{\partial}{\partial x}\right) d z-d z\left(\frac{\partial}{\partial x}\right) d y\right] \\
-(2 x+y+z) Y\left[d y\left(\frac{\partial}{\partial y}\right) d x-d x\left(\frac{\partial}{\partial y}\right) d y\right]-x Y\left[d x\left(\frac{\partial}{\partial y}\right) d y-d y\left(\frac{\partial}{\partial y}\right) d x\right] \\
+x Y\left[d x\left(\frac{\partial}{\partial y}\right) d z-d z\left(\frac{\partial}{\partial y}\right) d x\right]+x Y\left[d y\left(\frac{\partial}{\partial y}\right) d z-d z\left(\frac{\partial}{\partial y}\right) d y\right] \\
-(2 x+y+z) Z\left[d y\left(\frac{\partial}{\partial z}\right) d x-d x\left(\frac{\partial}{\partial z}\right) d y\right]-x Z\left[d x\left(\frac{\partial}{\partial z}\right) d y-d y\left(\frac{\partial}{\partial z}\right) d x\right] \\
+x Z\left[d x\left(\frac{\partial}{\partial z}\right) d z-d z\left(\frac{\partial}{\partial z}\right) d x\right]+x Z\left[d y\left(\frac{\partial}{\partial z}\right) d z-d z\left(\frac{\partial}{\partial z}\right) d y\right] \\
=-(2 x+y+z) X d y-x X d y+x X d z-(2 x+y+z) Y d x \\
+x Y d x+x Y d z-x Z d x-x Z d y
\end{gathered}
$$

Furthermore, the differential of Hamiltonian energy $H$ is obtained by

$$
d H=\frac{\partial H}{\partial x} d x+\frac{\partial H}{\partial y} d y+\frac{\partial H}{\partial z} d z
$$


If these Equations (23) are solved for $i_{X_{H}} \Phi=d H$

$$
\begin{aligned}
& X=\frac{1}{2(3 x+y+z)} \frac{\partial H}{\partial x}-\frac{1}{2(3 x+y+z)} \frac{\partial H}{\partial y}+\frac{1}{2 x} \frac{\partial H}{\partial z} \\
& Y=-\frac{1}{2(3 x+y+z)} \frac{\partial H}{\partial x}+\frac{1}{2(3 x+y+z)} \frac{\partial H}{\partial y}+\frac{1}{2 x} \frac{\partial H}{\partial z} \\
& Z=-\frac{1}{2 x} \frac{\partial H}{\partial x}-\frac{1}{2 x} \frac{\partial H}{\partial y}-\frac{(3 x+y+z)}{2 x^{2}} \frac{\partial H}{\partial z}
\end{aligned}
$$

From here

$$
\begin{aligned}
X_{H}= & \left(\frac{1}{2(3 x+y+z)} \frac{\partial H}{\partial x}-\frac{1}{2(3 x+y+z)} \frac{\partial H}{\partial y}+\frac{1}{2 x} \frac{\partial H}{\partial z}\right) d x \\
& +\left(-\frac{1}{2(3 x+y+z)} \frac{\partial H}{\partial x}+\frac{1}{2(3 x+y+z)} \frac{\partial H}{\partial y}+\frac{1}{2 x} \frac{\partial H}{\partial z}\right) d y \\
& +\left(-\frac{1}{2 x} \frac{\partial H}{\partial x}-\frac{1}{2 x} \frac{\partial H}{\partial y}-\frac{(3 x+y+z)}{2 x^{2}} \frac{\partial H}{\partial z}\right) d z
\end{aligned}
$$

Consider the curve and its velocity vector $\alpha: I \subset \mathbb{R} \rightarrow T^{*} M, \alpha(t)=\frac{\partial x}{\partial t}$ $d x+\frac{\partial y}{\partial t} d y+\frac{\partial z}{\partial t} d z$ such that an integral curve of the Hamiltonian vector field $X_{H}$, i.e., $X_{H}(\alpha(t))=\alpha(t), t \in I$. Then we find the following equations:

$$
\begin{gathered}
\left(\frac{1}{2(3 x+y+z)} \frac{\partial H}{\partial x}-\frac{1}{2(3 x+y+z)} \frac{\partial H}{\partial y}+\frac{1}{2 x} \frac{\partial H}{\partial z}\right) d x=\frac{\partial x}{\partial t} d x \\
\left(-\frac{1}{2(3 x+y+z)} \frac{\partial H}{\partial x}+\frac{1}{2(3 x+y+z)} \frac{\partial H}{\partial y}+\frac{1}{2 x} \frac{\partial H}{\partial z}\right) d y=\frac{\partial y}{\partial t} d y \\
\left(-\frac{1}{2 x} \frac{\partial H}{\partial x}-\frac{1}{2 x} \frac{\partial H}{\partial y}-\frac{(3 x+y+z)}{2 x^{2}} \frac{\partial H}{\partial z}\right) d z=\frac{\partial z}{\partial t} d z
\end{gathered}
$$

So, we find the following equations for motion: 


$$
\begin{aligned}
& \frac{\partial x}{\partial t}=\frac{1}{2(3 x+y+z)} \frac{\partial H}{\partial x}-\frac{1}{2(3 x+y+z)} \frac{\partial H}{\partial y}+\frac{1}{2 x} \frac{\partial H}{\partial z} \\
& \frac{\partial y}{\partial t}=-\frac{1}{2(3 x+y+z)} \frac{\partial H}{\partial x}+\frac{1}{2(3 x+y+z)} \frac{\partial H}{\partial y}+\frac{1}{2 x} \frac{\partial H}{\partial z} \\
& \frac{\partial z}{\partial t}=-\frac{1}{2 x} \frac{\partial H}{\partial x}-\frac{1}{2 x} \frac{\partial H}{\partial y}-\frac{(3 x+y+z)}{2 x^{2}} \frac{\partial H}{\partial z}
\end{aligned}
$$

Therefore, the equations introduced in (28) are called Hamilton equations on twistorial generalized Kähler manifolds and then the triple $\left(T^{*} M, \Phi, X_{H}\right)$ is thought to be a Hamiltonian mechanical system $\left(T^{*} M, g, J^{*}\right)$ on twistorial generalized Kähler manifolds with spacetime points.

\section{Equations Solving with Computer}

The location of each object in space represented with maximum three dimensions in physical space. These three dimensions can be labelled by a combination of three chosen from the terms time, position, mass, length, width, height, depth, density, and breadth. In this study, (28) equations are partial differential equations on twistorial generalized Kähler manifolds such that its solved with Maple computation program. Implicit solution (28) for $x(t)=t, y(t)=\sin (t), z(t)=\cos (t)$ is

$$
\begin{aligned}
& H(x, y, z, t)=-3 *\left(-1 / 6 * F_{1}(t)+1 / 6 * x\right) \\
& \quad * \cos (2 * t)-3 *\left(-1 / 6 * F_{1}(t)+1 / 6 * x\right) \\
& \quad * \sin (2 * t)-3 *\left(-F_{1}(t) * t+(x-1 / 3 * z) * t+1 / 3 * y\right) \\
& \quad * \cos (t)-3 *\left(1 / 3 * F_{1}(t) * t+(-1 / 3 * x-1 / 3 * y) * t+1 / 3 * y\right) \\
& \quad * \sin (t)+1 / 2 * F_{1}(t)-3 *(y-1 / 3 * z) * t-1 / 2 * x .
\end{aligned}
$$

As seen above (29) is a closed solution. To draw the graph of (29) must be made closed special preferences. 
Example 2. Special values of (28);

$$
\begin{aligned}
& H(x, y, z, t)=1 / 4 * P i-x-3 / 2 *\left(-1 / 16 * P i^{2}\right. \\
& +1 / 4 *(x-1 / 3 * z) * P i+1 / 3 * y) * 2^{(1 / 2)} \\
& -3 / 2 *\left(1 / 48 * P i^{2}+1 / 4 *(-1 / 3 * y-1 / 3 * x) * P i\right. \\
& +1 / 3 * y) * 2^{(1 / 2)}-3 / 4 *(-1 / 3 * z+y) * P i, t=\pi / 4
\end{aligned}
$$

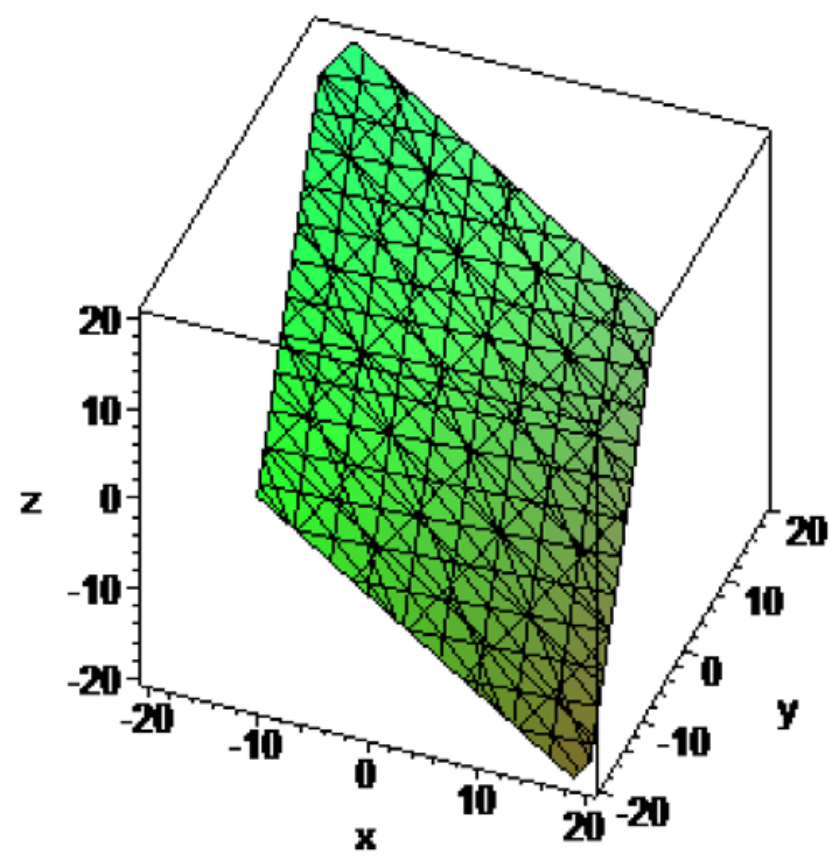

\section{Discussion}

A classical field theory explain the study of how one or more physical fields interact with matter which is used quantum and classical mechanics of physics branches. How the movement of objects in electrical, magnetically, and gravitational fields force is very important. Because, the classical theory of electromagnetism deals with electric and magnetic fields and their interaction with each other and with charges and currents. An electromagnetic field is a physical field produced by electrically charged objects and their locations over time. 
It is well-known that the motivation and one of the initial aims of twistor theory is to provide an adequate formalism for the union of quantum theory and general relativity. Twistor theory can also be used to solve non-linear differential equations, which are related to the selfduality equations that describe instantaneous in $\mathbb{R}^{4}$. In this study, the Hamilton mechanical equations for space-time points (28) derived on twistorial generalized Kähler manifolds may be suggested to deal with problems in electrical, magnetically, and gravitational fields for the path of movement (30) of defined space moving objects [21, 22].

\section{References}

[1] R. Penrose, Twistor algebra, J. Math. Phys. 8 (1967), 345-366.

[2] R. Penrose, Twistor Theory, its Aims and Achievements, C. J. Isham, R. Penrose, D. W. Sciama (Eds.), Proceedings of Oxford Symposium on Quantum Gravity, Clarendon Press, Oxford, (1975), 268-407.

[3] R. Pantilie, On a class of twistorial maps, Differential Geometry and its Applications 26 (2008), 366-376.

[4] S. Ianus, S. Marchiafava, L. Ornea and R. Pantilie, Twistorial maps between quaternionic manifolds, arXiv:0801.4587v2, (2008).

[5] S. Cecotti, A. Neitzke and C. Vafa, Twistorial topological strings and a $t t^{*}$ geometry for $N=2$ theories in 4d, arXiv:1412.4793v1, (2014).

[6] S. Santa-Cruz, Twistor geometry for hyper Kähler metrics on complex adjoint orbits, Annals of Global Analysis and Geometry 15 (1997), 361-377.

[7] R. Albuquerque, Twistorial constructions of special Riemannian manifolds.

[8] J. Davidov and O. Mushkarov, Twistor spaces of generalized complex structures, Journal of Geometry and Physics 56 (2006), 1623-1636.

[9] E. Loubeau and R. Pantilie, Harmonic morphisms between Weyl spaces and twistorial maps, Communications in Analysis and Geometry 14(5) (2006), 847-881.

[10] J. Davidov and O. Mushkarov, Twistorial construction of generalized Kähler manifolds, Journal of Geometry and Physics 57 (2007), 889-901.

[11] M. Dunajski, Twistor theory and differential equations, arXiv:0902.0274v2, (2009).

[12] S. Marchiafava, Twistorial maps between (para) quaternionic projective spaces, Bull. Math. Soc. Sci. Math. Roumanie Tome 52(100-3) (2009), 321-332.

[13] M. Tekkoyun, Mechanics systems on para-Kählerian manifolds of constant J-sectional curvature, http://arxiv.org/abs/0902.3569v1, (2009), 1-8. 
[14] N. Nowaczyk, J. Niediek and M. Firsching, Basics of Complex Manifolds, (2009).

[15] W. Ballmann, Lectures on Kähler Manifolds, ESI Lectures in Mathematics and Physics, 2006.

[16] D. J. Griffiths, Introduction to Electrodynamics, Prentice Hall, ISBN 0-13-805326-X, 1999.

[17] J. Klein, Escapes variationnels et mécanique, Ann. Inst. Fourier, Grenoble 12 (1962).

[18] A. Ibord, The geometry of dynamics, Extracta Mathematicable 11(1) (1996), 80-105.

[19] M. de Leon and P. R. Rodrigues, Methods of Differential Geometry in Analytical Mechanics, Elsevier Sc. Pub., 1989.

[20] R. Abraham, J. E. Marsden and T. Ratiu, Manifolds Tensor Analysis and Applications, Springer, 2001.

[21] B. Thidé, Electromagnetic field theory, (2012).

[22] R. G. Martín, Electromagnetic Field Theory for Physicists and Engineers: Fundamentals and Applications, Granada, 2007. 